\title{
Searches in Dilepton Final States at CDF
}

\author{
Andrew Ivanov \\ for the CDF Collaboration \\ Department of Physics, \\ University of California at Davis, \\ Davis, CA, 95616, USA \\ e-mail: andrew@fnal.gov
}

\begin{abstract}
Event signatures with two oppositely or same-sign charged leptons are predicted in many new physics scenarios. Such events could arise from chargino-neutralino production, in decays of pair produced supersymmetric top quarks, additional massive vector bosons or exotic heavy quarks. We present the most recent results from the CDF experiment on searches for physics beyond the Standard Model in events with two leptons in the final state produced in $p \bar{p}$ collisions at $\sqrt{s}=1.96 \mathrm{TeV}$ at Fermilab Tevatron.
\end{abstract}

Keywords: CDF, Tevatron, Supersymmetry

PACS: 12.60.-i, 12.60.Jv, 14.80.Ly

\section{INTRODUCTION}

We report on the most recent CDF results on searches for new physics in dilepton events. A review of searches for same-flavor dilepton resonances can be found elsewhere [1].

\section{SEARCHES FOR STOP QUARK}

Stop quark is a super-symmetric partner of the top quark. Due to the large top quark Yukawa coupling, the mass splitting between two stop quark mass eigen-states $\tilde{t}_{1}$ and $\tilde{t}_{2}$ may be large, allowing $\tilde{t}_{1}$ to be the lightest squark, that could be lighter than the top quark, and hence be accessible at Tevatron. In R-parity conserved SUSY models stop quarks are produced in pairs, and semi-leptonic stop decays would lead to event signatures with two oppositely charged leptons in the final state. CDF performed searches for stop quark considering two scenarios.

\section{Search for Stop $\tilde{t}_{1} \rightarrow b \tilde{\chi}_{1}^{ \pm} \rightarrow b \tilde{\chi}_{1}^{0} \ell v$}

In the first scenario $m_{\tilde{\chi}_{1}^{ \pm}}<m_{\tilde{t}_{1}}$, and $\tilde{\chi}_{1}^{0}$ is the lightest supersymmetric particle (LSP). In this case $\mathscr{B}\left(\tilde{t}_{1} \rightarrow b \tilde{\chi}_{1}^{ \pm}\right)=100 \%$, and leptonic $\tilde{\chi}_{1}^{ \pm}$decays lead to events signatures with two leptons, two $b$-jets and a large $E_{T}$ due to $v$ and $\tilde{\chi}_{1}^{0}$ escaping detection. These stops produce event signatures very similar to those from the top quark decays, and could potentially be hiding in samples of top quarks in the Tevatron data. 
B-Tagged Channel

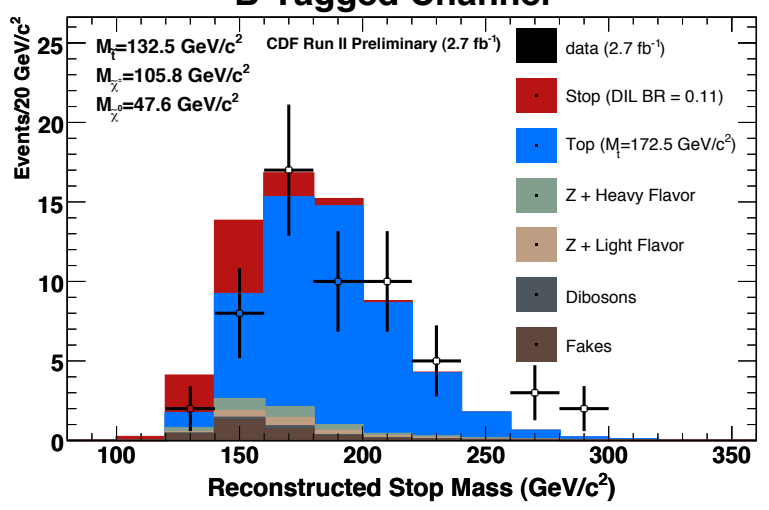

Observed $95 \% \mathrm{CL}$

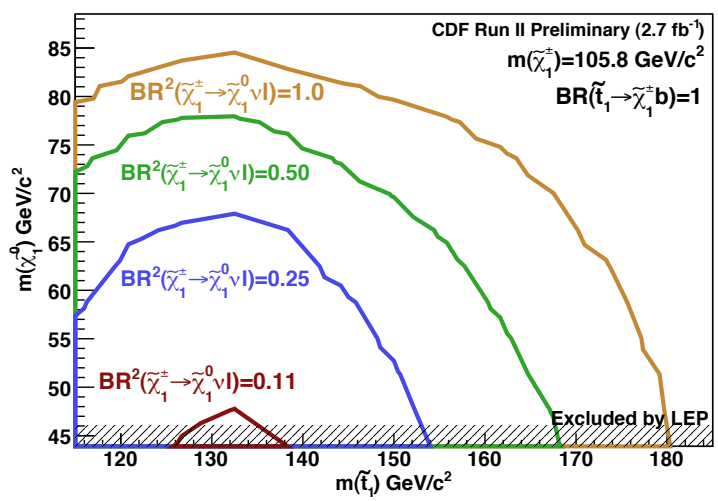

FIGURE 1. LEFT: The reconstructed stop quark mass distribution. RIGHT: The observed $95 \%$ C. L. exclusion regions in the $m_{\tilde{\chi}_{1}^{0}}$ and $m_{\tilde{t}_{1}}$ mass plane for several values of $\mathscr{B}\left(\tilde{\chi}_{1}^{ \pm} \rightarrow \tilde{\chi}_{1}^{0} \ell v\right)$. The excluded region corresponds to the area below the lines.

CDF divides stop candidate events into two categories: events with one of the jets identified as a $b$-jet ( $b$-tagged), and events with no $b$-jets. Next, it performs a full reconstruction of the stop quark mass in stop candidate events, the distribution of which for SM backgrounds and an example of stop signal is shown in Figure 1 (left). The data agrees with the SM alone, and no evidence for stop quark pair production is observed. These results are used to extract exclusion limits on the masses of $\tilde{t}_{1}, \tilde{\chi}_{1}^{0}$ and leptonic chargino branching ratio, which depends on the mass hierachy of super-symmetric particles, and shown in Figure 1 (right).

\section{Search for Stop $\tilde{t}_{1} \rightarrow b \ell \tilde{v}$}

The second scenario assumes that $\tilde{v}$ is the LSP (or $\tilde{v}$ decays invisibly to $v \tilde{\chi}_{1}^{0}$ or $v \tilde{G}$, where $\tilde{\chi}_{1}^{0}$ or $\tilde{G}$ is the LSP.) In this case the dilepton final state is produced through virtual $\tilde{\chi}_{1}^{ \pm}$or $\tilde{\ell}$. The event signature is similar to the previous search. However, this analysis focuses on lower lepton $p_{T}$ using dedicated dilepton triggers, and increases acceptance to a potential new physics signal by requiring one or more jets.

The search is performed as a set of counting experiments in several a priori selected signal regions. No significant deviation from the SM expectation is observed, and the results are used to extract exclusion limits in the $m_{\tilde{t}_{1}}-m_{\tilde{v}}$ plane under assumption that $\mathscr{B}\left(\tilde{t}_{1} \rightarrow b \ell \tilde{v}\right)=100 \%$, shown in Figure 2 (left).

\section{SEARCH FOR CHARGINO-NEUTRALINO $\tilde{\chi}_{1}^{ \pm} \tilde{\chi}_{2}^{0}$ PRODUCTION}

Chargino-neutralino production is mainly searched for, using trilepton signatures [2].However, if the mass splitting between $\tilde{\chi}_{1}^{ \pm}$and $\tilde{\chi}_{1}^{0}$, and between $\tilde{\chi}_{2}^{0}$ and $\tilde{\chi}_{1}^{0}$ is large, such that $W$ and $Z$ are produced on-shell, the leptonic branching ratios are 

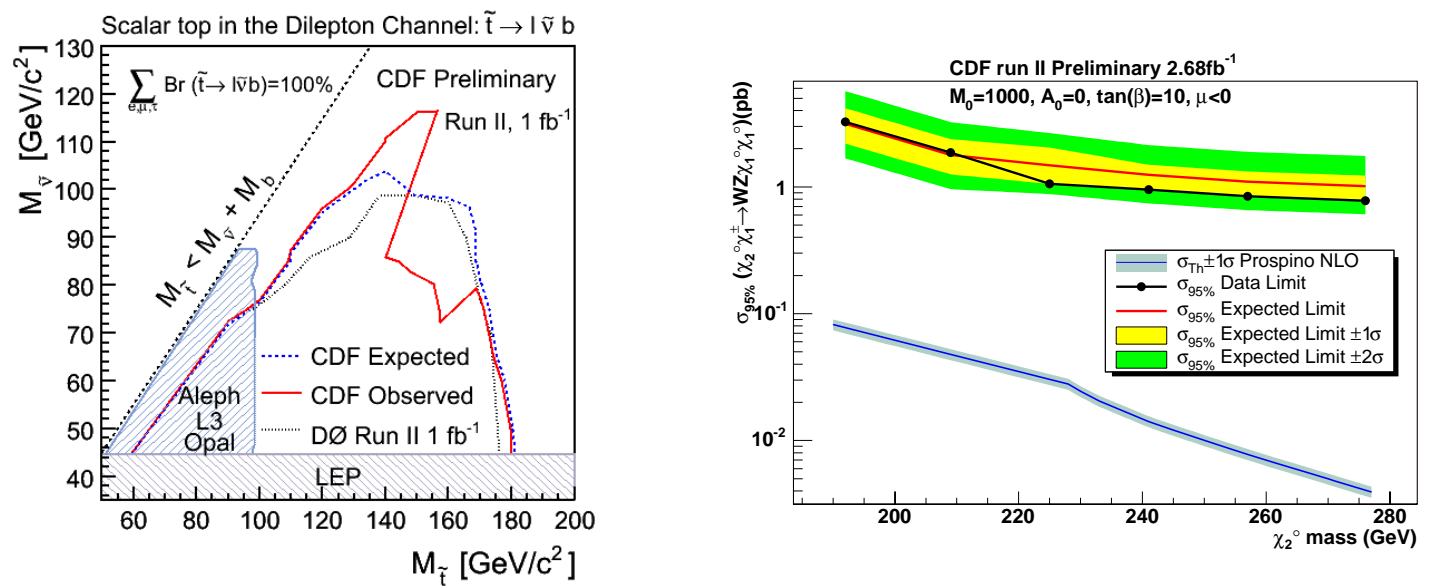

FIGURE 2. LEFT: The observed and expected limits in the $m_{\tilde{t}_{1}}-m_{\tilde{v}}$ plane. RIGHT: Cross section times branching ratio limits at $95 \% \mathrm{CL}$ for gaugino pair production.

suppressed. CDF performed a search for $\tilde{\chi}_{1}^{ \pm} \tilde{\chi}_{2}^{0} \rightarrow W \tilde{\chi}_{1}^{0} Z \tilde{\chi}_{1}^{0} \rightarrow q \bar{q} \tilde{\chi}_{1}^{0} \ell \bar{\ell} \tilde{\chi}_{1}^{0}$, assuming that $Z$ decays leptonically and $W$ decays into a pair of hadrons. The analysis requires two leptons and at least two jets and imposes invariant mass constraints on a pair of leptons and a pair of jets to be consistent with $Z$ and $W$ pole masses respectively. The search is performed as a counting experiment in the region of high- $E_{T}$. In the data sample corresponding to $\sim 3 \mathrm{fb}^{-1}$ of luminosity, 7 events are observed, while $6.4 \pm 0.9$ are expected. The obtained results are used to extract the limit on $\tilde{\chi}_{1}^{ \pm} \tilde{\chi}_{2}^{0}$ production cross section, shown in Figure 2 (right).

\section{SEARCH FOR SCALAR NEUTRINO $\tilde{v}_{\tau}$}

If R-parity is violated, SUSY processes can result in lepton and baryon number violation. In this case single-produced $\tilde{v}_{\tau}$ decaying into a pair of leptons of different flavor $(e \mu, e \tau$ or $\mu \tau)$ can lead to a striking resonant peak in the spectrum of the dilepton invariant mass.

CDF searched for $\tilde{v}_{\tau}$ production in three dilepton channels simultaneously. The $\tau$ leptons are identified via their hadronic decays. Due to $v$ from $\tau$ decay escaping detection, the "visible" four-momentum of a $\tau$ candidate is obtained from summing the four-momenta of charged tracks and the energies from neutral particles pointing to a calorimeter cluster, and is used in forming the dilepton invariant mass. The mass spectrum in the $e \tau$ channel is shown in Figure 3 (left).

The dilepton mass distribution is scanned for several different mass points and using the Bayesian approach is converted into the $95 \% \mathrm{C}$. L. limit on the $\sigma(p \bar{p} \rightarrow \tilde{v}) \times \mathscr{B}(\tilde{v} \rightarrow$ $\ell \ell)$. For a chosen set of RPV couplings $\left(\lambda_{311}^{\prime}=0.10, \lambda_{132}=0.05, \lambda_{133}=0.05, \lambda_{231}=\right.$ $0.05)$ the exclusion limits on the mass of $\tilde{v}_{\tau}$ are $482 \mathrm{GeV} / \mathrm{c}^{2}, 475 \mathrm{GeV} / \mathrm{c}^{2}$ and $556 \mathrm{GeV} / \mathrm{c}^{2}$ in the $e \tau, \mu \tau$ and $e \mu$ channels respectively. 

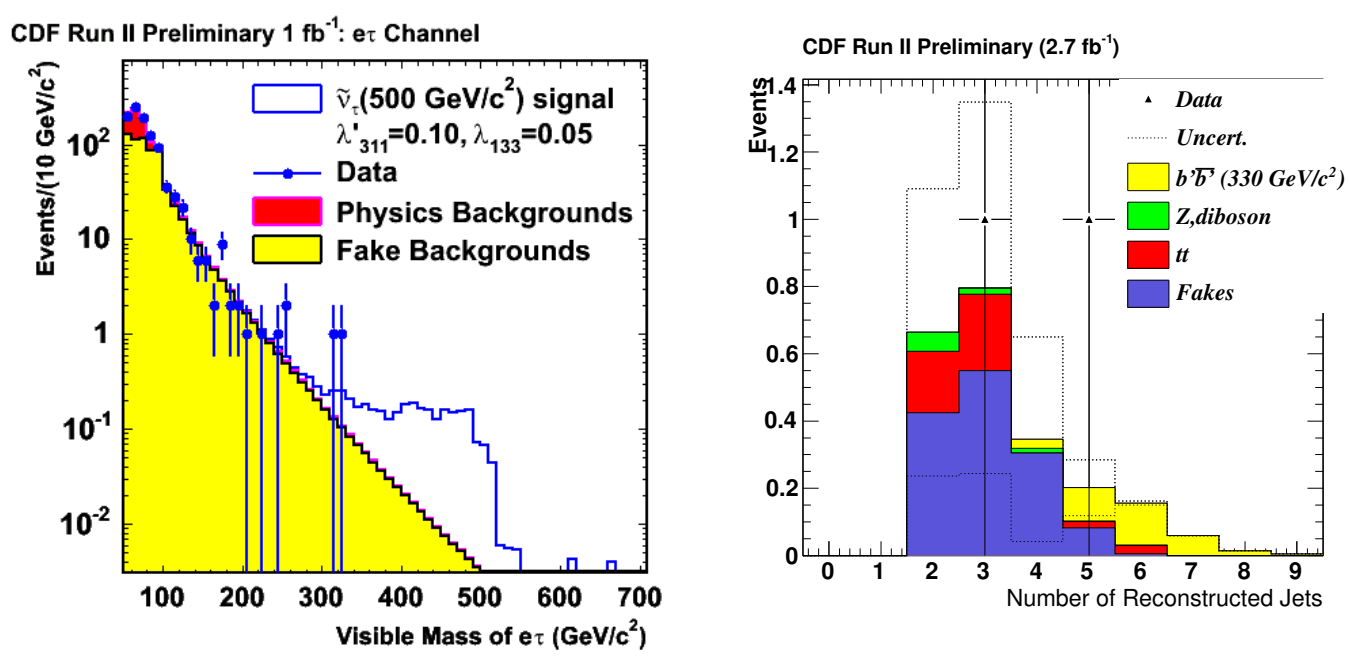

FIGURE 3. LEFT: Visible mass of $e \tau$. RIGHT: Jet multiplicity distribution in same-sign dilepton events.

\section{SEARCH FOR FOURTH-GENERATION DOWN-TYPE QUARK $b^{\prime}$}

The fourth generation has been historically disfavored, although remains consistent with electroweak precision data [3]. Present bounds on the mass of $b^{\prime}$ imply that $m_{b^{\prime}}$ is above $\sim 255 \mathrm{GeV} / \mathrm{c}^{2}$ [4], and therefore, if exists, it should primarily decay into $t W$. Pair-produced $b^{\prime}$ is expected to have four $W$ in the final state. CDF searches for these events using same-sign dilepton events. Charge-misidentification rate is very small, and the main background comes from jets misidentified as leptons. Hadronically decaying $W$ bosons produce additional jet activity, hence the jet multiplicity distribution serves as a discriminating variable between SM backgrounds and a potential $b^{\prime}$ signal.

The jet multiplicity spectrum is presented in Figure 3 (right), and by performing the likelihood fit to this distribution CDF obtains 95\% C. .L lower limit on the $b^{\prime}$ mass at 338 $\mathrm{GeV} / \mathrm{c}^{2}$. The same procedure is employed to search for composite fermions of charge $5 / 3$ decaying into $t W$, and CDF excludes such objects with masses below $365 \mathrm{GeV} / \mathrm{c}^{2}$.

\section{CONCLUSIONS}

In this article the recent results on searches for new physics in the dilepton final states at $\mathrm{CDF}$ are presented. No evidence for new physics has been observed and stringent exclusion limits are set on the masses of new particles.

\section{REFERENCES}

1. M. Gold, "Searches for new massive vector bosons with CDF", these proceedings.

2. J. Strologas, "Search for trilepton SUSY signal at CDF", these proceedings.

3. G. D. Kribs, T. Plehn, M. Spannowsky, and T. M. P. Tait, Phys. Rev. D 76, 075016 (2007).

4. T. Aaltonen et al., The CDF Collaboration, Phys. Rev. D 76, 072006 (2007). 